\title{
вмJ Global Health Geographic barriers to establishing a successful hospital referral system in rural Madagascar
}

\author{
Felana Angella Ihantamalala, ${ }^{1,2}$ Matthew H Bonds, ${ }^{2,3}$ Mauricianot Randriamihaja, ${ }^{3}$ \\ Luc Rakotonirina, ${ }^{3}$ Vincent Herbreteau, ${ }^{4}$ Christophe Révillion, ${ }^{5}$ \\ Serge Rakotoarimanana, ${ }^{3}$ Giovanna Cowley, ${ }^{3}$ Tsirinomen'ny Aina Andriatiana, ${ }^{3}$ \\ Alishya Mayfield, ${ }^{2,3}$ Michael L Rich, ${ }^{2,3,6}$ Rado J L Rakotonanahary, ${ }^{3}$ \\ Karen E Finnegan, ${ }^{3}$ Andriamanolohaja Ramarson, ${ }^{3}$ Benedicte Razafinjato, ${ }^{3}$ \\ Bruno Ramiandrisoa, ${ }^{7}$ Andriamihaja Randrianambinina, ${ }^{8}$ Laura F Cordier, ${ }^{3}$ \\ Andres Garchitorena (i) ${ }^{3,9}$
}

To cite: Ihantamalala FA, Bonds MH, Randriamihaja M, et al. Geographic barriers to establishing a successful hospital referral system in rural Madagascar. BMJ Global Health 2021;6:e007145. doi:10.1136/ bmjgh-2021-007145

Handling editor Seye Abimbola

- Additional material is published online only. To view please visit the journal online (http://dx.doi.org/10.1136/ bmjgh-2021-007145).

Received 9 August 2021 Accepted 17 November 2021

Check for updates

C Author(s) (or their employer(s)) 2021. Re-use permitted under CC BY-NC. No commercial re-use. See rights and permissions. Published by BMJ.

For numbered affiliations see end of article.

Correspondence to

Dr Felana Angella Ihantamalala; felana.angella@gmail.com

\section{ABSTRACT}

Background The provision of emergency and hospital care has become an integral part of the global vision for universal health coverage. To strengthen secondary care systems, we need to accurately understand the time necessary for populations to reach a hospital. The goal of this study was to develop methods that accurately estimate referral and prehospital time for rural districts in low and middle-income countries. We used these estimates to assess how local geography can limit the impact of a strengthened referral programme in a rural district of Madagascar.

Methods We developed a database containing: travel speed by foot and motorised vehicles in Ifanadiana district; a full mapping of all roads, footpaths and households; and remotely sensed data on terrain, land cover and climatic characteristics. We used this information to calibrate estimates of referral and prehospital time based on the shortest route algorithms and statistical models of local travel speed. We predict the impact on referral numbers of strategies aimed at reducing referral time for underserved populations via generalised linear mixed models.

Results About $10 \%$ of the population lived less than 2 hours from the hospital, and more than half lived over 4 hours away, with variable access depending on climatic conditions. Only the four health centres located near the paved road had referral times to the hospital within 1 hour. Referral time remained the main barrier limiting the number of referrals despite health system strengthening efforts. The addition of two new referral centres is estimated to triple the population living within 2 hours from a centre with better emergency care capacity and nearly double the number of expected referrals.

Conclusion This study demonstrates how adapting geographic accessibility modelling methods to local scales can occur through improving the precision of travel time estimates and pairing them with data on health facility use.

\section{Key questions}

What is already known?

- In rural areas, geographic access to care is a key determinant of hospital use, which tends to fall exponentially as distance to health facilities increases.

- Eighty per cent of the population should live within 2 hours of a hospital in case of emergency.

- A variety of methods exist for estimating population time to reach the nearest hospital; these tend to differ in precision depending on the study setting (high vs low-income countries)

What are the new findings?

- This study demonstrates a new method to estimate key measures of geographic accessibility to hospital care that are context specific and highly accurate.

- Only $10 \%$ of our study population lived within 2 hours of a district hospital, which is much lower than average estimates for sub-Saharan Africa.

- We found that geographic barriers leading to increased referral times remained the primary barrier limiting the number of referrals completed by health centres to the district hospital in a rural low-income country.

What do the new findings imply?

- Highly precise methods to estimate travel time can be adapted for use at local scales, and are especially useful for rural areas of the developing world.

- The results from this method can inform health actors on ways to redesign the local health system to overcome existing barriers to care and improve overall population health.

\section{INTRODUCTION}

The provision of emergency care has recently become an integral part of the global vision for universal health coverage (UHC) as a way to ensure the 'timely and effective delivery of life-saving health care services to those in 
need'. ${ }^{1}$ While primary care services remain the cornerstone of any health system, it is estimated that nearly half of all deaths and about a third of disability-adjusted life years in low and middle-income countries (LMICs) could be addressed by well-functioning emergency care systems. ${ }^{2}$ The 2019 World Health Assembly urged member states to strengthen emergency care systems by creating policies, governance and funding mechanisms that promote the inclusion of emergency care into national health strategies. ${ }^{1}$ These systems should overcome the multiple geographical, financial and social barriers that limit the access of patients in LMICs to hospital care..$^{3-7}$ In rural areas specifically, where healthcare infrastructure is sparse, geographic access to care is a key determinant of hospital use, which tends to fall exponentially as the travel time to health facilities increases. ${ }^{8-10}$ To improve geographic access to hospital care, the Lancet Commission on Global Surgery proposed that at least $80 \%$ of a country's population should be within 2 hours of a facility with essential surgery and anaesthetic services. ${ }^{11}$

A variety of methods exist for estimating population time to reach the nearest hospital; these tend to differ according to the study setting. In high-income countries, where road infrastructure and emergency services are well developed, travel time is typically estimated using the shortest route estimations, with assumptions about vehicle travel speeds. ${ }^{12-15}$ To improve on these methods, estimates are often compared or calibrated using surveys of referred patients, ${ }^{12-14}$ trip data from emergency service records ${ }^{13}$ or ambulance global positioning system (GPS) measurements. ${ }^{16} 17$ In contrast, studies in rural areas of low-income countries combine estimates of travel time by foot and motorised vehicles to reflect local realities in hospital accessibility, as road infrastructure is deficient and ambulance services are rarely available. However, since much of the geographic information on roads and paths that people use to reach hospitals in these areas is still missing, the prevailing technique for estimating travel time is the use of friction surfaces based on terrain and land cover characteristics, together with available road networks. ${ }^{8}$ 18-21

Friction surface algorithms are widely used and the basis of 'AccessMod', a free tool developed by the WHO to increase the adoption of geographic accessibility analyses into health planning. ${ }^{22}{ }^{23}$ Using such methods, researchers have estimated that over $70 \%{ }^{24}$ and as much as $92 \%^{25}$ of the population in sub-Saharan Africa live within 2 hours of a hospital. However, methods that rely on friction surfaces represent only a 'best guess' of the routes people use, with simplifying assumptions that rarely account for the impact of context-specific factors on travel speeds or on accessibility at the local level. Recent studies are increasingly including context-specific factors via expert consultations to improve geographic accessibility estimates at the national level,,${ }^{27}$ but these remain absent in continental studies. As a result, current estimates of hospital accessibility in sub-Saharan Africa could be grossly overestimated.
To help increase access to timely emergency and hospital care in LMICs, health system strengthening efforts need to integrate emergency care training, processes and resources at different levels of the health system. ${ }^{11}$ The WHO recommends that patients be transferred from primary health centres to referral hospitals by ambulances in emergency cases. ${ }^{28}$ In practice, formal emergency care systems in LMICs are rare and poorly implemented due to the lack of resources (skilled personnel, infrastructure, transport) to ensure timely provision to emergency services. ${ }^{29}$ Even where ambulances are available, time delays in hospital transfers limit the coverage and effectiveness of emergency services, ${ }^{48}$ increasing the risk of death for children, ${ }^{30}$ pregnant women with delivery complications ${ }^{31}$ or patients with conditions such as stroke ${ }^{32}$ or asthma. ${ }^{33}$ Given the importance of ensuring timely access to emergency and hospital care, more accurate estimations of referral and prehospital time are key to identify global needs and funding gaps and to improve the basic design of health systems at the national and local levels in LMICs.

The goal of this study was to develop methods that accurately estimate referral and prehospital time for rural health districts in LMICs, based on locally calibrated models and context-specific information. We used these estimates to assess how local geography can limit the impact of a strengthened referral programme and which additional strategies can help overcome geographic barriers. This study was conducted in Ifanadiana district, in Southeastern Madagascar, which has been established as a model district for UHC by the government of Madagascar, implemented in partnership with the healthcare non-governmental organisation (NGO) PIVOT (see the 'Study area and intervention' section). Ifanadiana district has one of the only rural ambulance referral systems in the country. We build on previous work in which we improved the precision of geographic accessibility estimates to primary care, showing that standard methods tend to overestimate the proportion of the population with access to care. ${ }^{34}$ We hypothesised that estimates of hospital access might be subject to a similar or greater overestimation, and that even small differences in time to access hospital care can have substantial impacts on the use of emergency services.

To address this, we first developed a database containing the movements of people and motorised vehicles in Ifanadiana district over several months as well as a full mapping of all roads, footpaths and households in the district. We used this information to calibrate estimates of referral and prehospital time based on shortest route algorithms and statistical models of local travel speed. Finally, we studied the impact of referral time on the evolution of the number of referrals completed by each health centre in the district using model estimates to predict the impact on referral numbers of strategies aimed at reducing referral time for underserved populations. 


\section{METHODS}

\section{Study area and intervention}

Ifanadiana is a rural district located in Southeastern Madagascar. Ifanadiana has 15 communes and 195 fokontany (smallest administrative boundary comprising one or several villages). The district health system includes one referral hospital and 21 health centres, including a major primary care health centre (Centre de Santé de Base niveau 2, CSB2) in each commune, and six additional basic health centres (Centre de Santé de Base niveau 1, CSB1) in some of the larger communes, which provide a more limited range of primary care services. In addition, two community health workers per fokontany provide care for common illnesses of children under 5 years. The Ministry of Public Health (MoPH)-PIVOT model district is based on strengthening the health system at all three levels of care, ensuring (1) health system readiness via the availability of sufficient health personnel, essential medicines, necessary equipment and infrastructure renovations; (2) the provision of high-quality accessible care, including improved diagnostic capacity, clinical programmes, infection control, psychosocial assistance, the removal of user fees and the distribution of social kits to the patients; and (3) integrated data systems at all levels of care to allow for robust monitoring, evaluation and research on the intervention. More information about the health system strengthening intervention can be found in the online supplemental table S1.

To improve access to emergency and hospital care, the MoPH-PIVOT partnership created an ambulance referral system, which operates 24 hours/day and 7 days/week. This service includes three fully equipped ambulances with 12 trained paramedics, and processes to ensure care continuity. For instance, all health centres in the district have been equipped with cellphones and a call centre has been established at the district hospital for specialised guidance and to coordinate referrals. ${ }^{35}$ Patients requiring secondary care are transported from accessible health centres or meeting points to the district hospital, and those who need more specialised care are transported to the university hospital in the city of Fianarantsoa (2 hours away by car) or to specialised facilities in Antananarivo (10 hours away by car). In non-emergency cases needing secondary care, other means of transport can be used by patients (such as local bus: 'taxi brousse') and transportation fees are reimbursed by PIVOT.

Multiple geographic barriers limit the success of this referral programme. The district is made up of deep valleys and a mountainous landscape, with an altitude gradient ranging from 1000 to $100 \mathrm{~m}$ from West to East. Road infrastructure in the district is scarce (figure 1), with a single paved road that runs across the district from East to West through four of its communes. In addition, there are two main non-paved roads connecting the district capital, Ifanadiana, with the communes in the North and South. Only a fraction of these roads are accessible by allterrain vehicles, especially under rainy conditions, and the availability of such vehicles is very limited. The rest of the road network consists of smaller paths accessible by all-terrain motorcycles, with most of the network accessible only by foot. To further improve access to emergency and secondary care, the MoPH-PIVOT partnership is planning to turn two major health centres in the North and South of the district into referral centres that will be

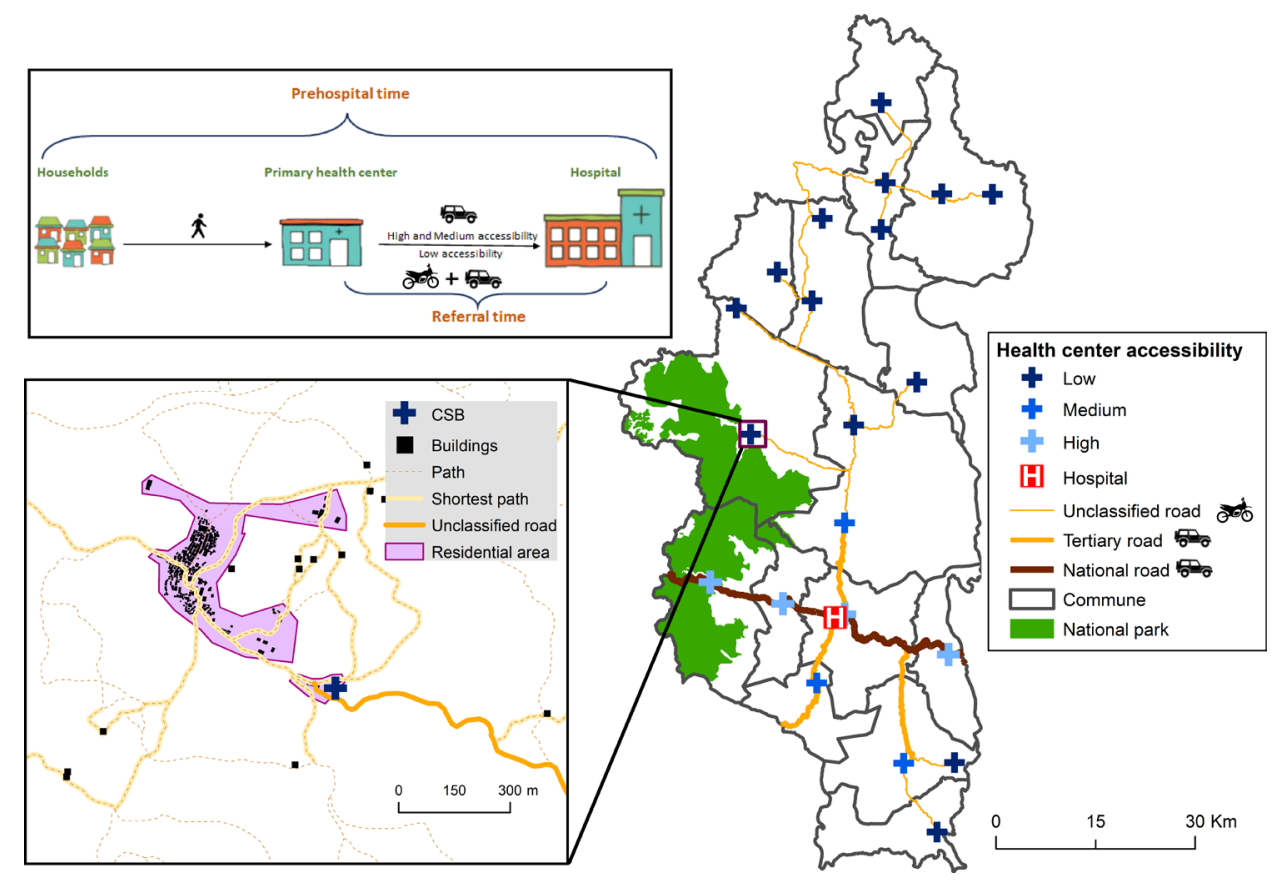

Figure 1 Prehospital circuit in Ifanadiana district. A map of the health system and road network in Ifanadiana. Top inset panel displays the means of transportation patients generally use to reach the district hospital in case of referral, according to geographic accessibility and level of care. Bottom inset panel shows the level of resolution in the OpenStreetMap (OSM) database to precisely estimate patient routes. CSB, primary care health centre (Centre de Santé de Base in French) 
able to provide acute care for patients in distress. This research was done in support of this referral programme to accurately estimate current accessibility to secondary care and to assess the potential impact of this strategy at addressing existing challenges.

\section{Data collection}

Road and footpath network in Ifanadiana district

To accurately estimate prehospital time from each household in Ifanadiana district to the referral hospital, we first used the OpenStreetMap (OSM) ${ }^{36}$ platform to map the full network of roads and footpaths, as well as all residential areas, houses and rice fields in the district. This was done in collaboration with the Humanitarian OpenStreetMap Team, ${ }^{37}$ which allowed us to divide the district into several thousand $1 \mathrm{~km}^{2}$ tiles that were then mapped by individual users using very-high-resolution satellite images. The mapping of each tile was done in two steps: first, a user mapped all geographic entities and then another user validated the mapping to ensure data quality. As a result, over $20000 \mathrm{~km}$ of footpaths and over 100000 buildings were mapped. The purpose of obtaining this data set was to determine the shortest path between each household and its nearest health centre, and between each health centre and the district hospital, which emulates the process of a referral from the community to the hospital (figure 1). Distance estimations were done via the Open Source Routing Machine tool, which uses the Dijkstra's routing algorithm, searching iteratively the shortest path from a single node (eg, the household) to the destination node in a network (eg, the health centre). The algorithm does not consider terrain or land cover through friction surfaces; instead, this information is integrated afterwards on the shortest paths via statistical models of travel speed (next section). This work is extensively described in Ihantamalala $e t a l$ 's study.

Travel speed under different terrains and conditions for people and motorised vehicles

To obtain context-specific estimates of travel speed under different local terrain and climatic conditions, we collected two types of data sets. First, to estimate travel speeds by foot, from September 2018 to April 2019, we recorded the movements of different individuals (NGO staff and community health workers) in a representative sample of footpaths in Ifanadiana district. For this, we used the Android V.3.0.2 of the OsmAnd app ${ }^{38}$ installed in Samsung Tab A10 tablets using a record frequency of 10 s. For each GPS point, the following data were recorded: geographical location, date, time and altitude. Overall, 168 different routes were recorded, representing nearly $1000 \mathrm{~km}$ of footpaths. This data set was used to estimate the time it takes for patients to reach the nearest health centre prior to referral, and is described in further detail in Ihantamalala $e t a l$ s study.

Second, to estimate travel speeds for motorised vehicles (cars and motorcycles), we obtained trip recordings of all the NGO cars and motorcycles from TAG-IP, ${ }^{39}$ a company that provides vehicle GPS data collection and management services in Madagascar. All the NGO motorised vehicles are equipped with one of three types of GPS: Careu U1, Careu Uw1 or FMB204. These GPS devices were calibrated to record location every $10 \mathrm{~s}$ and data were transferred and stocked directly into the server of TAG-IP. For this project, we obtained a sample of data from $17 \mathrm{March}$ to 5 May 2019 and from 1 February to 4 April 2020. A total of $77709 \mathrm{~km}$ of motorised vehicle routes from Ifanadiana district were collected, $61258 \mathrm{~km}$ from cars and $16451 \mathrm{~km}$ from motorcycles (see online supplemental table S2). For each GPS point, the following data were recorded: identity of the car/motorcycle, coordinates of their position, date, time and altitude. This data set was then used to estimate the referral time of patients from each health centre to the district hospital.

\section{Satellite imagery and remote sensing}

We obtained elevation and precipitation data from remotely sensed data. We downloaded the Shuttle Radar Topography Mission digital elevation model (DEM) from the US Geological Survey, ${ }^{40}$ which gives elevation with a $30 \mathrm{~m}$ ground resolution. We also acquired precipitation estimates from the National Aeronautics and Space Administration (NASA) Prediction of Worldwide Energy Resources project, ${ }^{41}$ with a spatial resolution of $0.5^{\circ} \times 0.5^{\circ}$.

\section{Referral data}

The MoPH-PIVOT partnership collected hospital data on the number of patients referred from health centres to the hospital, with data accessible via the NGO's dashboard $^{42}$ for use by programme managers. We obtained data from PIVOT's monitoring and evaluation team for all referrals completed from January 2014 to July 2020. The data included the aggregated number of referrals per month, means of transportation used and the name of the health centre from which the referral originated. A referral was considered complete when the patient reached the hospital and feedback was provided to the referring health centre. Personal identifiable information from all referral data was removed to protect patient privacy. Use of health system data for this study was approved by the medical inspector of Ifanadiana district and by Harvard's Institutional Review Board.

\section{Data analysis}

Estimation of referral time from health centres and households to the district hospital

This work builds on previous work where we estimated the travel time from every household in Ifanadiana district to the nearest health centre. ${ }^{34}$ Here we estimate total prehospital time for a referral by adding the time from each health centre to the district hospital. To calculate the referral time between health centres and the hospital, we used the database of the NGO's vehicle movements and divided each trip into $100 \mathrm{~m}$ segments which we intersected with geographic and climatic data. We explored the impact on travel speed of: the type of 
motorised vehicle, the type of road, rainfall conditions (1 month average of precipitation, in millimetres), driving through residential areas, crossing bridges and the distance since the trip began (see online supplemental table S3). Initially, we carried out exploratory and univariate analyses to understand the relationship between travel speed and each variable of interest, transforming explanatory variables where necessary. Then, we carried out a multivariate analysis using a Poisson mixedeffects model that included all of the above variables as fixed effects and each separate trip as a random intercept. Based on this model, we predicted the referral time for each of the 21 health centres according to the particular route characteristics to reach the hospital, and two types of rainfall conditions: dry and rainy conditions. For the predictions under rainy conditions, we used the median value of rainfall recorded during the vehicle trips.

Second, we combined travel time from health centres to the hospital with the travel time from households to health centres estimated in our previous research. ${ }^{34}$ In brief, travel time to the nearest health centre was estimated using the same methods as described above, where a statistical model of travel speed by foot was first built from field data as a function of land cover, climatic and elevation characteristics. Then, predictions were made for all of the 41426 shortest routes from each household or residential area (group of households) in the district to the nearest health centre under both dry and rainy conditions. Our estimates of prehospital time assume that a patient went to the closest health centre by foot, and was then transferred via ambulance if the health centre was accessible by car, and if not, by other means of transport (in our case by motorcycle) to an ambulance pick-up point (figure 1). Ambulances and cars can drive on the paved road, and $17 \mathrm{~km}$ North and $16 \mathrm{~km}$ South of the paved road on non-paved roads (figure 1). These assumptions are based on the reports by patients and healthcare providers in Ifanadiana.

Finally, we aggregated individual household prehospital times to estimate the percentage of the population at different categories of access to the district hospital.

Comparison of prehospital time results with existing methods

To assess how the locally calibrated estimates obtained in our study compared with other methods commonly used in geographical accessibility modelling of hospital care, we estimated referral and prehospital time using simplified assumptions generally accepted for similar settings. For referral time, we used the same shortest route, but with a simplified assumption that vehicles travel at a speed of $50 \mathrm{~km} /$ hour on paved secondary roads and 30 $\mathrm{km} /$ hour on non-paved roads. For estimates of prehospital time, we used the module 'accessibility analysis' of the software AccessMod V.5, which is based on friction surface algorithms, together with the district's DEM, OSM road network and land cover data sets, where walking speed values for each class of land cover and path, and motorised speed values for road networks were assigned following travel scenarios for AccessMod V.3.0 from Ray and Ebener ${ }^{22}$ and Ouma $e t a l^{24}$ (see online supplemental table S4). Then, the Zonal Statistics tool of ArcGIS was used to get the percentage of population per travel time class. Travel speeds by motorised vehicles in AccessMod were not affected by terrain slopes.

Assessment of determinants of monthly referrals by health centres We assessed the evolution of the number of referrals completed by health centres since the beginning of the referral programme to understand the impact of geographic barriers. For this, we first classified health centres into four categories according to their geographic accessibility: high for those on the paved road $(n=4)$, medium for those accessible by ambulance on a nonpaved road $(n=3)$ and low for those not accessible by ambulance and requiring additional means of transportation $(n=14)$. The latter were further subdivided into major $(\mathrm{CSB} 2, \mathrm{n}=8)$ or basic health centres (CSB1, $\mathrm{n}=6$ ). We explored the number of referrals completed and the means of transportation used for each category of geographic accessibility.

Second, we used multivariate statistical models to study how geographic factors affected the monthly number of referrals completed by each health centre, adjusting for relevant variables. We assessed the impact of geographic factors via orthogonal polynomial terms of degree 2 (exponential, non-linear relationship) for the travel time between the health centre and the district hospital, under both rainy and dry conditions, including the categories of access described above. We adjusted the model for health system factors, such as the type of health facility (CSB1 or CSB2) and whether user fees at the primary care level had been removed (binary variable). In addition, we adjusted for time-varying trends, such as the annual increase in the number of referrals, seasonal trends (using a sinus function with a period of 12 months) and the lagged number of referrals for each health centre (lag of 1 month).

We fit a negative binomial mixed-effects model with all the above variables as fixed effects, and each health centre as a random intercept. Model selection was carried out via stepwise selection whereby a multivariate model was first considered including all variables with a $p$ value below 0.1 in univariate analyses. Then, a reduced model was obtained by keeping only those variables significantly associated with the response variable (ie, $\mathrm{p}$ values below $0.05)$. All statistical analyses were carried out using the 'Ime4' package in R statistical software V.3.6.2. ${ }^{43}$

\section{Predictions of estimated impact for strategies aimed at reducing} referral time to secondary care

Given geographic challenges to transport patients from health centres in the North and the South of the district to the district hospital, plans are in place to equip one health centre in each area with the necessary infrastructure, equipment, training and human resources to become referral centres. The choice of these two 
particular health centres depended on operational and programmatic factors (existing infrastructure, acceptability and support from district officers and staff, etc) so optimising geographic accessibility was not the only criterion considered. To assess the impact of this strategy, we first estimated health centre referral times and household prehospital times under a scenario in which health centres refer patients to the closest referral facility among these two new referral centres and the district hospital. Then, using the statistical model of the number of monthly referrals by health centre described above (excluding lagged referrals), we predicted how reductions in referral time would affect the number of referrals completed in the future. For this, we predicted the number of referrals for a period of 2 years from the end of the study (July 2020 to June 2022) from the fixed effects of the model using referral times for a scenario with and without the two new centres, and we estimated the difference in the number of referrals between the two scenarios for health centres according to geographic accessibility.

\section{RESULTS}

Distribution and local determinants of referral and prehospital times in Ifanadiana district

Multivariate analyses of over $70000 \mathrm{~km}$ of motorised trips by NGO vehicles in Ifanadiana district revealed that motorised vehicles in the district drove at about 60 $\mathrm{km} /$ hour on the paved national road (table 1). Speeds decreased by over half on non-paved roads (tertiary or unclassified), and motorcycles drove slightly faster than cars. Travel speed was nearly one-third slower when crossing a bridge or through a residential area. In addition, travel speed was reduced in driving conditions under rain (less than half for every change in a unit of the $\log _{10}$ of average monthly rainfall) or on a slope (onethird slower for every change in a unit of the $\log _{10}$ of $\%$ of absolute slope change).

Predictions of travel time from this model revealed that only the four health centres located near the paved road had referral times to the hospital within approximately 1 hour under both dry and rainy conditions (table 2). Two additional health centres reachable by non-paved roads had referral times of 1 hour under dry conditions, but these increased to about 1 hour 30 min under rainy conditions. The 14 health centres with low accessibility in the North and South of the district required a motorcycle ride or other means of transportation for $20-100 \mathrm{~km}$, plus an ambulance ride for the remaining $17 \mathrm{~km}$ to the hospital. Minimum referral time for these health centres varied from 1 hour 43 min to over 6 hours under dry conditions and from 2 hours 23 min to over 8 hours under rainy conditions. These context-specific, datadriven estimates of referral time are significantly higher than when using common values of travel speed $(50 \mathrm{~km} /$ hour on paved roads and $30 \mathrm{~km} /$ hour on non-paved roads). For instance, referral times for low-accessibility
Table 1 Multivariate analysis of local factors affecting travel speed by motorised vehicles (Poisson linear mixed models, with individual track as random intercept)

\begin{tabular}{|c|c|c|}
\hline & Estimate* $^{*}$ & $95 \% \mathrm{Cl}$ \\
\hline Intercept (km/hour) & 59.98 & 58.15 to 61.86 \\
\hline \multicolumn{3}{|l|}{ Type of road } \\
\hline National road (paved) & (Ref) & \\
\hline $\begin{array}{l}\text { Tertiary road (non- } \\
\text { paved) }\end{array}$ & 0.45 & 0.45 to 0.45 \\
\hline $\begin{array}{l}\text { Unclassified (non- } \\
\text { paved) }\end{array}$ & 0.46 & 0.45 to 0.46 \\
\hline \multicolumn{3}{|l|}{ Type of vehicle } \\
\hline Car/ambulance & (Ref) & \\
\hline Motorcycle & 1.15 & 1.13 to 1.17 \\
\hline \multicolumn{3}{|l|}{ Residential area } \\
\hline No & (Ref) & \\
\hline Yes & 0.67 & 0.67 to 0.67 \\
\hline \multicolumn{3}{|l|}{ Bridge } \\
\hline No & (Ref) & \\
\hline Yes & 0.70 & 0.69 to 0.7 \\
\hline \multicolumn{3}{|l|}{ Distance since trip started } \\
\hline Within $2 \mathrm{~km}$ & (Ref) & \\
\hline Over 2 km & 1.18 & 1.18 to 1.19 \\
\hline Slope $\left(\%, \log _{10}\right)$ & 0.68 & 0.68 to 0.68 \\
\hline Rainfall $\left(\mathrm{mm}, \log _{10}\right)$ & 0.49 & 0.47 to 0.5 \\
\hline
\end{tabular}

*Model coefficients and Cls are exponentiated to reflect incidence rate ratio (relative change in average speed per unit change in the explanatory variable), except for the intercept which reflects absolute value in kilometre per hour.

health centres were underestimated by a minimum of 30 min and a maximum of 1 hour 45 min as compared with our model (table 2).

Combining estimates of referral time by motorised vehicles with previous estimates of walking time from each household to the nearest health centre allowed us to estimate the minimum time necessary to seek care at the hospital, or prehospital time, at the population level. We found that minimum prehospital time varied from 5 min to 10 hours under dry conditions and from $8 \mathrm{~min}$ to 14 hours under rainy conditions. Only $13 \%$ of the population lived less than 2 hours from the hospital and $48 \%$ lived within 4 hours (table 3 ). This percentage decreased further under rainy conditions, where only $9 \%$ and $33 \%$ of the population can reach the hospital within 2 and 4 hours, respectively. More than half of the population need over 4 hours to reach the hospital. As with referral time, comparison of these estimates with commonly used methods for geographic accessibility modelling (friction surfaces, AccessMod) revealed a substantial overestimation of the population with appropriate access to the hospital. For instance, friction surface estimates suggested that nearly $30 \%$ of the population could reach 
Table 2 Predictions of minimum referral time from each health centre to the district hospital under different climatic conditions and predictions with a simple assumption of $50 \mathrm{~km} /$ hour on paved roads and $30 \mathrm{~km} /$ hour on non-paved roads

\begin{tabular}{|c|c|c|c|c|c|}
\hline \multirow[b]{2}{*}{ Health centres } & \multirow[b]{2}{*}{ Accessibility } & \multirow[b]{2}{*}{ Distance (km) } & \multicolumn{3}{|l|}{ Referral time } \\
\hline & & & Dry conditions & $\begin{array}{l}\text { Rainy } \\
\text { conditions }\end{array}$ & $\begin{array}{l}\text { Simplified } \\
\text { assumption }\end{array}$ \\
\hline CSB2 Antaretra & High & 28 (vehicle) & $48 \mathrm{~min}$ & 1 hour 6 min & $34 \mathrm{~min}$ \\
\hline CSB2 Ifanadiana & & 2 (vehicle) & $5 \min$ & $7 \min$ & $2 \min$ \\
\hline CSB2 Kelilalina & & 9 (vehicle) & $15 \mathrm{~min}$ & $20 \mathrm{~min}$ & $11 \mathrm{~min}$ \\
\hline CSB2 Ranomafana & & 22 (vehicle) & $33 \mathrm{~min}$ & $46 \mathrm{~min}$ & $26 \min$ \\
\hline CSB2 Ambiabe & Medium & 14 (vehicle) & 1 hour 7 min & 1 hour $33 \mathrm{~min}$ & $28 \mathrm{~min}$ \\
\hline CSB2 Androrangavola & & 17 (vehicle), 23 (motorcycle) & 1 hour 43 min & 2 hours $23 \mathrm{~min}$ & 1 hour 6 min \\
\hline CSB2 Tsaratanana & & 17 (vehicle) & $57 \mathrm{~min}$ & 1 hour 20 min & $34 \mathrm{~min}$ \\
\hline $\begin{array}{l}\text { CSB2 Ambohimanga } \\
\text { du Sud }\end{array}$ & Low CSB2 & 17 (vehicle), 50 (motorcycle) & 3 hours $15 \mathrm{~min}$ & 4 hours $31 \mathrm{~min}$ & 2 hours $13 \mathrm{~min}$ \\
\hline CSB2 Ambohimiera & & 17 (vehicle), 28 (motorcycle) & 2 hours $14 \mathrm{~min}$ & 3 hours 6 min & 1 hour 29 min \\
\hline CSB2 Ampasinambo & & 17 (vehicle), 110 (motorcycle) & 5 hours $59 \mathrm{~min}$ & 8 hours $19 \mathrm{~min}$ & 4 hours $13 \mathrm{~min}$ \\
\hline CSB2 Analampasina & & 17 (vehicle), 59 (motorcycle) & 3 hours $41 \mathrm{~min}$ & 5 hours $6 \mathrm{~min}$ & 2 hours $31 \mathrm{~min}$ \\
\hline CSB2 Atsindra & & 17 (vehicle), 38 (motorcycle) & 2 hours $42 \min$ & 3 hours $45 \mathrm{~min}$ & 1 hour $49 \min$ \\
\hline CSB2 Fasintsara & & 17 (vehicle), 94 (motorcycle) & 5 hours $15 \mathrm{~min}$ & 7 hours $17 \mathrm{~min}$ & 3 hours $41 \mathrm{~min}$ \\
\hline CSB2 Maroharatra & & 17 (vehicle), 105 (motorcycle) & 5 hours $45 \mathrm{~min}$ & 7 hours $59 \mathrm{~min}$ & 4 hours $3 \mathrm{~min}$ \\
\hline CSB2 Marotoko & & 17 (vehicle), 31 (motorcycle) & 2 hours $28 \mathrm{~min}$ & 3 hours $25 \mathrm{~min}$ & 1 hour 34 min \\
\hline CSB1 Ambalavolo & Low CSB1 & 17 (vehicle), 114 (motorcycle) & 6 hours $14 \mathrm{~min}$ & 8 hours $39 \mathrm{~min}$ & 4 hours $21 \mathrm{~min}$ \\
\hline CSB1 AmbodiaraSud & & 17 (vehicle), 103 (motorcycle) & 5 hours $43 \mathrm{~min}$ & 7 hours $55 \mathrm{~min}$ & 3 hours $59 \mathrm{~min}$ \\
\hline $\begin{array}{l}\text { CSB1 Ambodimanga } \\
\text { Nord }\end{array}$ & & 17 (vehicle), 67 (motorcycle) & 4 hours $7 \mathrm{~min}$ & 5 hours $43 \mathrm{~min}$ & 2 hours $47 \mathrm{~min}$ \\
\hline CSB1 Analamarina & & 17 (vehicle), 20 (motorcycle) & 1 hour $52 \min$ & 2 hours $35 \mathrm{~min}$ & 1 hour 13 min \\
\hline CSB1 Mahasoa & & 17 (vehicle), 29 (motorcycle) & 2 hours $3 \mathrm{~min}$ & 2 hours $50 \mathrm{~min}$ & 1 hour $18 \mathrm{~min}$ \\
\hline CSB1 Maromanana & & 17 (vehicle), 52 (motorcycle) & 3 hours 22 min & 4 hours $40 \mathrm{~min}$ & 2 hours $17 \mathrm{~min}$ \\
\hline
\end{tabular}

CSB, Primary health care centre (Centre de Santé de Base in French).

the hospital within 2 hours under dry conditions, and $21.4 \%$ under rainy conditions.

\section{Impact of geographic accessibility on the number of hospital referrals}

A total of 8464 hospital referrals were completed from January 2014 to July 2020. Referral patterns were driven by health centre accessibility, with over three-quarters of patients referred from the four health centres with high accessibility, and less than $10 \%$ of patients referred from the 14 health centres with low accessibility (figure 2, see online supplemental table S5). The most common means of transportation was by ambulance $(40.83 \%)$, followed by taxi brousse $(20.14 \%)$ and walking $(18.93 \%)$. However, the means of transportation varied significantly according to health centre accessibility. In health centres with high and medium accessibility more than two-thirds of referrals were by ambulance or taxi brousse, while referrals from health centres with low accessibility required other

Table 3 Distribution of the population in Ifanadiana according to their time to reach the district hospital (prehospital time)

\begin{tabular}{|c|c|c|c|c|}
\hline \multirow[b]{3}{*}{ Prehospital time (hours) } & \multicolumn{4}{|l|}{ Population (\%) } \\
\hline & \multicolumn{2}{|c|}{ Estimation using statistical model } & \multicolumn{2}{|c|}{ Estimation using AccessMod } \\
\hline & Dry conditions & Rainy conditions & Dry conditions & Rainy conditions \\
\hline$(0,2)$ & 12.95 & 9.30 & 29.28 & 21.40 \\
\hline$(2,4)$ & 35.39 & 23.74 & 26.88 & 25.34 \\
\hline$(4,6)$ & 31.13 & 29.28 & 23.26 & 19.82 \\
\hline$(6,8)$ & 14.65 & 19.68 & 8.92 & 15.03 \\
\hline$>8$ & 5.88 & 18 & 11.65 & 18.40 \\
\hline
\end{tabular}



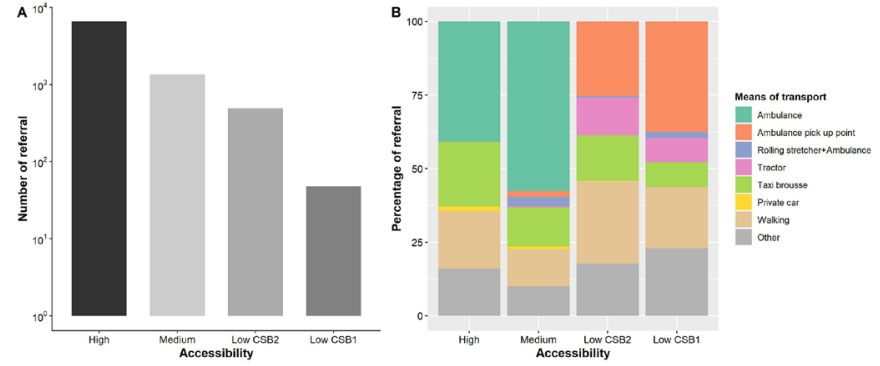

Figure 2 Patterns observed for hospital referrals in Ifanadiana according to geographic accessibility of health centres, 2014-2020. (A) Total number of referrals completed during the study period for each category of health centre accessibility ( $y$-axis in logarithmic scale). (B) Means of referral transport used for each category of health centre accessibility. CSB, primary care health centre (Centre de Santé de Base in French)

means (figure 2). About one-quarter of referrals from low-accessibility facilities required the patient to travel tens of kilometres to a point $17 \mathrm{~km}$ from the hospital for pick-up by the ambulance, another quarter of referrals were completed by foot and many were completed by tractor or rolling stretchers (figure 2).

The number of referrals increased more than threefold during the study period, from an average of 34 referrals per month in 2014 to 116 referrals per month in the first 7 months of 2020 (figure 3). Referral time to the hospital remained the most important determinant of the monthly
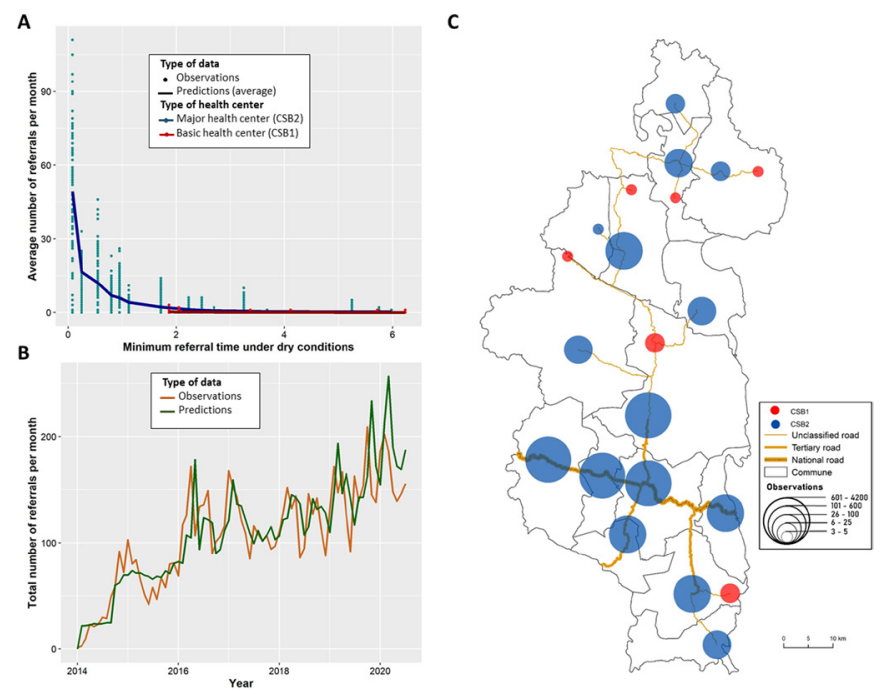

Figure 3 Observed and predicted temporal and spatial trends for hospital referrals in Ifanadiana district, 2014-2020. (A) Average number of referrals completed by health centres each month as a function of the minimum referral time from the health centre to the hospital, stratified by type of health centre. (B) Evolution of the total number of referrals per month observed (brown line) and predicted (green line) over the study period. (C) Spatial distribution of hospital referrals by health centre (blue circles $=\mathrm{CSB} 2$ and red circles $=\mathrm{CSB} 1$ ), with larger circle size corresponding to a higher number of referrals completed by the health centre. CSB, primary care health centre (Centre de Santé de Base in French)

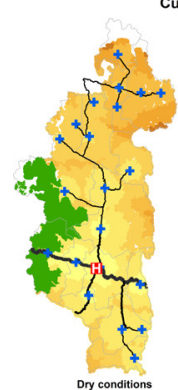

Current situation

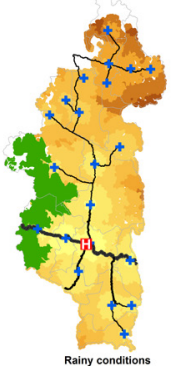

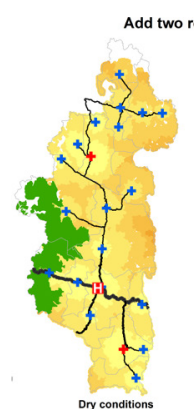

Add two referral centers

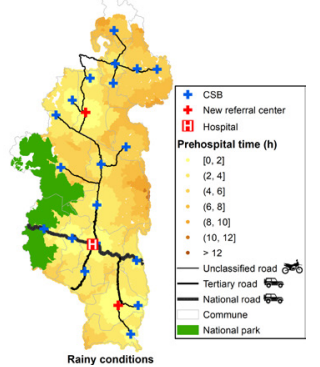

Figure 4 Distribution of the minimum prehospital time in Ifanadiana district under different scenarios. Each map shows point estimates for travel time from each of the 41426 households and residential areas in Ifanadiana to the district hospital (current situation) and under a scenario where two additional referral centres are added (red crosses). Estimates are provided under dry conditions (without rainfall) and rainy conditions (with rainfall). Shading from yellow to brown represents categories of increasing prehospital time. In blank are areas where no household or residential area was observed and therefore no point estimates of travel speed were available. CSB, primary care health centre (Centre de Santé de Base in French)

number of referrals completed by health centres, even after adjusting for other relevant variables (see online supplemental table S4). We observed an exponential decrease in the number of patients referred as a function of referral time, especially within the first 2 hours (figure 3). Other factors that were statistically associated with an increase in the number of referrals were the size of the health centre (major vs basic health centre), the removal of user fees at the health centre level and time-varying trends (annual increase and lagged number of referrals).

\section{Potential impact of strategies that reduce referral time to secondary care}

Predictions using locally calibrated models described above suggested that the implementation of two additional referral centres would lead to a significant increase in access to secondary care, both in terms of a reduction of prehospital time and an increase in the number of referrals completed (figures 4 and 5). The main improvements in terms of prehospital time would be for the whole Southern and Northern parts of the district, where most populations could access a referral centre within 4 hours regardless of climatic conditions (figure 4). This is in contrast to the current situation, in which large geographical areas, especially in the North, have prehospital times of over 6 hours under dry conditions and over 8 hours under rainy conditions (figure 4). The proportion of the population within 2 hours of a centre with higher acute care capacity would increase by nearly threefold from $12.95 \%$ to $30.56 \%$ under dry conditions and from $9.30 \%$ to $23.19 \%$ under rainy conditions (figure 4 ). The proportion of the population needing over 4 hours to reach a health facility would more than halve, decreasing from $51.66 \%$ to $24.77 \%$ under dry conditions and from $66.96 \%$ to $38.16 \%$ under rainy conditions. 
A

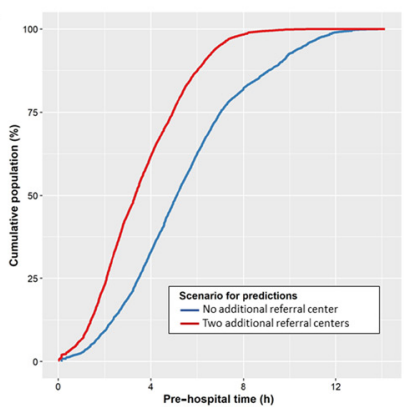

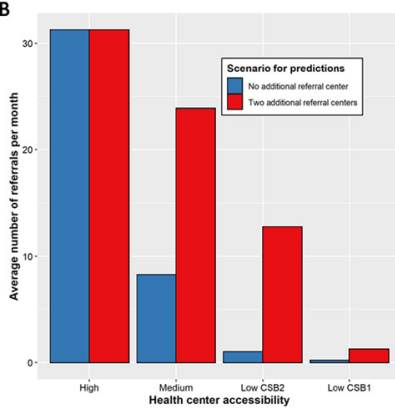

Figure 5 Potential improvements in accessibility to secondary care by adding two referral centres. (A) Changes in population-level prehospital time in a scenario with only one referral hospital (blue) and the addition of two referral centres (red) under rainy conditions. (B) Changes in the average number of monthly referrals completed by health centres with different levels of geographic accessibility. CSB, primary care health centre (Centre de Santé de Base in French)

Predictions of the number of referrals for the period from July 2020 to June 2022 suggested that this strategy would lead to a twofold increase in the number of referrals, from an average of 1906 referrals per year predicted in a scenario with only the district hospital to 3666 referrals per year in a scenario with two new referral centres (figure 5). This increase would disproportionately benefit less accessible health centres over high-accessible health centres. While the number of referrals would remain unchanged for health centres with high accessibility at about 30 referrals per month, those with medium accessibility would increase from 8 to 24 referrals per month. For health centres with low accessibility, major health centres (CSB2) would increase monthly referrals from 1 to 13 and basic health centres (CSB1) would increase referrals from 0 to 1 per month.

\section{DISCUSSION}

In order to address the burden of mortality and disability in LMICs, ensuring access to emergency and hospital care for all has become a global priority, as part of a broader movement to achieve UHC. But many health systems are not designed to reach everyone. As the time necessary to reach a hospital is one of the main determinants to accessing care, it is important to have an accurate understanding of who is able to access service. In this study, we combine GPS data of people and motorised vehicles, a full district mapping and remote sensing analyses to calibrate statistical models of local referral and prehospital time in a rural district of Madagascar. We showed that only about $10 \%$ of the population live within 2 hours of the district hospital and more than half live over 4 hours away, with variable access depending on climatic conditions. Despite health system strengthening efforts in place that have more than tripled the number of hospital referrals between 2014 and 2020, we found that geographic barriers leading to increased referral

times remained the primary barrier limiting the number of referrals completed by health centres.

Estimates of geographic accessibility to hospital care for Ifanadiana district were significantly lower than those reported in previous studies. For instance, multicountry studies using friction surface methods combined with available geographic information system (GIS) data had estimated that over $70 \%^{24}$ and as much as $92 \%{ }^{25}$ of the population in sub-Saharan Africa live within 2 hours of a hospital. Importantly, the scale of these analyses requires trade-offs in the resolution of available input data (eg, resampling at $1 \mathrm{~km} \times 1 \mathrm{~km}$ ) and in the assumptions used (eg, travel speed for routes and terrains) that likely impact the precision of their travel time estimates. We show here that using simplified assumptions of travel speed for estimating referral time or methods relying on friction surfaces for estimating prehospital time in our district led to a substantial underestimation of the travel time necessary to reach the hospital and to an overestimation of the population living within 2 hours of the hospital. If these local results are representative of larger trends for sub-Saharan Africa, it suggests that results from multicountry studies should be taken with caution. For example, a recent continental study suggested that all regions in Madagascar had over $80 \%$ of their population living within 2 hours of a hospital, ${ }^{44}$ which is in stark contrast with the results we obtained here for Ifanadiana. This has major implications for international health policies attempting to reach the recommended goal of ensuring that $80 \%$ of the population can access a hospital within 2 hours, since this threshold may be harder to reach than previously anticipated.

Our study is consistent with previous evidence showing that geographic accessibility to hospital care has a very important role in use. ${ }^{45} 46$ Moreover, it reveals that geographic accessibility remains a key barrier even where health system strengthening efforts exist to improve access to hospital care. The MoPH-PIVOT partnership in Ifanadiana included a rare 24/7 ambulance referral system, processes to ensure care continuity such as an emergency call centre and the removal of user fees for referred patients. Yet, over three-quarters of hospital referrals in the district came from the four health centres (out of 21) located near the only paved road in the district and within 1 hour of the hospital. This is consistent with a similar study in Tanzania, where $85.6 \%$ of referrals came from highly accessible primary healthcare centres located within 1 hour of the hospital. ${ }^{47}$ These results reinforce the need to reduce the referral time from health centres to hospitals in LMICs as an effective way to increase hospital use, revealing the limits of health system strengthening programmes without further collaboration with other sectors outside the health system. Indeed, improving the quality of local roads, which is beyond the scope of healthcare NGOs or ministries of health, could significantly expand the geographic reach of ambulances and lead to substantial increases in the number of referrals in addition to having other economic benefits. 
We used our models to provide more information on the impact of solutions within the capacity of the MoPH-PIVOT partnership. Previous studies have used geographic accessibility modelling to assess the optimal location of new facilities in order to minimise population travel time. ${ }^{20} 4849$ For instance, in Ethiopia, researchers found that upgrading a few strategically located facilities and reconfiguring referral networks could increase the percentage of population served within 2 hours from $70 \%$ to $90 \%$, and reduce the average travel time by nearly half. ${ }^{20}$ Another study in Kenya suggested that 32 additional facilities would be necessary to reach UHC for maternity health services in one of Kenya's rural counties. ${ }^{48}$ In this study, we characterised both referral time and its relationship with the number of hospital referrals completed by health centres, which allowed us to inform programme managers on the potential impact of a strategy they are planning to implement (ie, adding two referral centres) on prehospital time at the population level and on the number of expected referrals for each health centre. It is important to note that the goal was not to identify the two health centres that optimised geographic access since the choice had already been made according to operational and programmatic factors, but the methods developed here could inform alternative scale-up strategies in the future. We found that this strategy could be highly effective, doubling the number of hospital referrals in Ifanadiana district, with a notable threefold increase in referrals for health centres of medium geographic accessibility and more than a 10 -fold increase in referrals for health centres with low geographic accessibility. In parallel, the proportion of the district population within 2 hours from a centre with surgical capabilities would nearly triple.

This study had several limitations. First, many scenarios can be considered for how local populations reach a hospital. We assumed that people go to the closest health centre by foot and are then referred to the hospital by motorised vehicle because that best reflects local practices in Ifanadiana. However, patients could travel to the hospital by foot, which would increase prehospital time (significantly in some cases), or they could come directly to the hospital without passing through the primary health centre, which would reduce prehospital time. Analyses of referral data showed that less than $20 \%$ of referred patients arrived at the hospital by foot, while the fee exemption programme (eg, costs of referrals are covered for patients sent from a health centre to the hospital) in combination with the long distances to reach the hospital strongly incentivised patients to consult at primary health centres first. Therefore, these alternative scenarios probably had a limited impact on the results presented here. Second, predictions of the impact of adding two referral centres on the number of referrals in 2020-2022 were based on trends observed during 20142020. If other factors affecting referrals, such as road conditions or health system practices, were to change significantly between these two periods, this could bias our predictions. However, we are not aware that any such changes have taken place to date in our study area. Third, the lack of available data from local weather stations in our area meant that we had to use global interpolated rainfall estimates with a coarse resolution $\left(0.5^{\circ}\right)$ to parametrise travel speeds, which could have led to some inaccuracies in our travel speed model. Finally, as with any local study, the generalisability of results presented here may be limited to other rural, low-income settings with similar characteristics as Ifanadiana in terms of terrain, road infrastructure and health system capacity. Our travel speed model was calibrated with information from Ifanadiana district, which would be directly transferable to districts in the East of Madagascar. Importantly, the wide heterogeneity of landscapes, terrains and road conditions captured in our data set suggests that effect coefficients and speed estimates could be applicable to most rural districts in Madagascar, although this needs to be validated. In addition, further research is needed to assess whether similar trends in hospital geographic access and use are observed in other areas of sub-Saharan Africa.

A national scale-up of the methods presented here could be useful to ministries of health and many other health actors strengthening public health systems in developing countries, but this requires investments to expand available information on OSM in order to gain the required level of detail, especially in rural areas. We are currently scaling these comprehensive OSM mapping and geographic accessibility models to several districts in Southeastern Madagascar with support from the United States Agency for International Development (USAID), which could potentially inform the resources necessary for a national scale-up. Recent developments in artificial intelligence, such as the Maxar-Digital Globe building footprints or Facebook's RapiD tool for road networks, could greatly accelerate mapping in low-resource settings, but in our experience substantial human mapping is still necessary for it to be of high quality and publicly available to everyone.

\section{CONCLUSION}

This study demonstrates a new method to estimate key measures of geographic accessibility to hospital care that are context specific and highly accurate. Using highresolution GIS data and local fieldwork, these models can be calibrated to better reflect local practices and barriers in accessing hospital care. Comparisons of results with other commonly used methods in geographic accessibility modelling suggest that estimates for sub-Saharan Africa may systematically overestimate the percentage of the population living within 2 hours of a hospital. More broadly, we show how using geographic accessibility modelling methods adapted to local scales, by improving the precision of travel time estimates and pairing them with health facility utilisation data, can inform health actors on ways of redesigning the local health system to 
overcome existing barriers to care and improve overall population health.

\section{Author affiliations}

${ }^{1}$ Research, NGO PIVOT, Ifanadiana, Fianarantsoa, Madagascar

${ }^{2}$ Department of Global Health and Social Medicine, Harvard Medical School, Boston, Massachusetts, USA

${ }^{3} \mathrm{NGO}$ PIVOT, Ranomafana, Madagascar

${ }^{4}$ Espace-Dev, IRD, Université des Antilles, Université de Guyane, Université de Montpellier, Université de La Réunion, Phnom Penh, Cambodia

${ }^{5}$ Espace-Dev, IRD, Université des Antilles, Université de Guyane, Université de Montpellier, Université de La Réunion, Saint-Pierre, France

${ }^{6}$ Division of Global Health Equity, Brigham and Women's Hospital, Boston,

Massachusetts, USA

${ }^{7}$ Ministry of Public Health, Antananarivo, Madagascar

${ }^{8}$ Ministere de la Sante Publique, Antananarivo, Madagascar

${ }^{9}$ MIVEGEC, Université de Montpellier, CNRS, IRD, Montpellier, France

Acknowledgements We would like to thank all OSM contributors to the cartography of Ifanadiana district, apart from the authors of this article, and in particular the OpenStreetMap Associations of La Réunion and Madagascar for the mapping parties they organised and their individual contributions. We would also like to thank Mrs Tina Razermera of TAG-IP for the motorised vehicle data. In addition, we would like to thank Community Health team staff at PIVOT and community health workers for helping us to collect fieldwork data and PIVOT's Monitoring and Evaluation staff for the referral data.

Contributors FAI, MHB, LR and AG conceived and designed the study. MHB, VH, $\mathrm{CR}, \mathrm{LFC}$ and $\mathrm{AG}$ advised on the analysis. FAl, MR and $A G$ analysed the data. FAl, $\mathrm{VH}, \mathrm{CR}, \mathrm{MR}$ and $\mathrm{AG}$ contributed to the interpretation of the data. FAl and $A G$ are the responsible for the overall content of this manuscript. FAI, MHB, MR, LR, VH, CR, SR, GC, TAA, AM, MLR, RJLR, KEF, ARam, BRaz, BRam, ARan, LFC and AG drafted the manuscript. All authors read and approved the final manuscript.

Funding This work was supported by a grant from the Institut de Recherche pour le Développement (Project IRD Coup de Pouce 'MAGIE') and internal funding from PIVOT.

Map disclaimer The inclusion of any map (including the depiction of any boundaries therein), or of any geographic or locational reference, does not imply the expression of any opinion whatsoever on the part of BMJ concerning the legal status of any country, territory, jurisdiction or area or of its authorities. Any such expression remains solely that of the relevant source and is not endorsed by BMJ. Maps are provided without any warranty of any kind, either express or implied.

Competing interests None declared.

Patient consent for publication Not required.

Provenance and peer review Not commissioned; externally peer reviewed.

Data availability statement Data are available in a public, open access repository. Data are available upon reasonable request. GIS data on roads, footpaths and buildings in Ifanadiana district are publicly available on OpenStreetMap (https://www.openstreetmap.org) and on the Shinny app described in this study (http://research.pivot-dashboard.org:3838/). Health system data may be shared upon request to the address research@pivotworks.org.

Supplemental material This content has been supplied by the author(s). It has not been vetted by BMJ Publishing Group Limited (BMJ) and may not have been peer-reviewed. Any opinions or recommendations discussed are solely those of the author(s) and are not endorsed by BMJ. BMJ disclaims all liability and responsibility arising from any reliance placed on the content. Where the content includes any translated material, BMJ does not warrant the accuracy and reliability of the translations (including but not limited to local regulations, clinical guidelines, terminology, drug names and drug dosages), and is not responsible for any error and/or omissions arising from translation and adaptation or otherwise.

Open access This is an open access article distributed in accordance with the Creative Commons Attribution Non Commercial (CC BY-NC 4.0) license, which permits others to distribute, remix, adapt, build upon this work non-commercially, and license their derivative works on different terms, provided the original work is properly cited, appropriate credit is given, any changes made indicated, and the use is non-commercial. See: http://creativecommons.org/licenses/by-nc/4.0/.
ORCID iD

Andres Garchitorena http://orcid.org/0000-0001-6225-5226

\section{REFERENCES}

1 Seventy-second World Health Assembly. Emergency care systems for universal health coverage: ensuring timely care for the acutely ill and injured, 2019.

2 Kobusingye OC, Hyder AA, Bishai D. Chapter 68. Emergency Medical Services. In: Disease Control Priorities in Developing Countries. 2nd Edition, 2006: 1261-80.

3 Kobusingye OC, Hyder AA, Bishai D, et al. Emergency medical systems in low- and middle-income countries: recommendations for action. Bull World Health Organ 2005;83:626-31.

4 Rocha $\mathrm{TAH}$, da Silva NC, Amaral PV, et al. Addressing geographic access barriers to emergency care services: a national ecologic study of hospitals in Brazil. Int J Equity Health 2017;16:149.

5 Richard F, Witter S, de Brouwere V. Innovative approaches to reducing financial barriers to obstetric care in low-income countries. Am J Public Health 2010;100:1845-52.

6 Moomba K, Van Wyk B. Social and economic barriers to adherence among patients at Livingstone General Hospital in Zambia. Afr J Prim Health Care Fam Med 2019;11:e1-6.

7 Cannoodt L, Mock C, Bucagu M. Identifying barriers to emergency care services. Int J Health Plann Manage 2012;27:e104-20.

8 Blanford JI, Kumar S, Luo W, et al. It's a long, long walk: accessibility to hospitals, maternity and integrated health centers in niger. Int J Health Geogr 2012;11:24.

9 Alegana VA, Khazenzi C, Akech SO, et al. Estimating Hospital catchments from in-patient admission records: a spatial statistical approach applied to malaria. Sci Rep 2020;10:1324.

10 Kelly C, Hulme C, Farragher T, et al. Are differences in travel time or distance to healthcare for adults in global North countries associated with an impact on health outcomes? A systematic review. BMJ Open 2016;6:e013059.

11 Meara JG, Leather AJM, Hagander L, et al. Global surgery 2030: evidence and solutions for achieving health, welfare, and economic development. Lancet 2015;386:569-624.

12 Haynes R, Jones AP, Sauerzapf V, et al. Validation of travel times to hospital estimated by GIS. Int J Health Geogr 2006;5:40.

13 Patel AB, Waters NM, Blanchard IE, et al. A validation of ground ambulance pre-hospital times modeled using geographic information systems. Int J Health Geogr 2012;11:42.

14 Nallamothu BK, Bates ER, Wang Y, et al. Driving times and distances to hospitals with percutaneous coronary intervention in the United States: implications for prehospital triage of patients with STelevation myocardial infarction. Circulation 2006;113:1189-95.

15 Klein MB, Kramer CB, Nelson J, et al. Geographic access to burn center hospitals. JAMA 2009;302:1-7.

16 Budge S, Ingolfsson A, Zerom D. Empirical analysis of ambulance travel times: the case of Calgary emergency medical services. Manage Sci 2010;56:716-23.

17 Westgate BS, Woodard DB, Matteson DS, et al. Large-network travel time distribution estimation for ambulances. Eur J Oper Res 2016;252:322-33.

18 Makanga PT, Schuurman N, von Dadelszen P, et al. A scoping review of geographic information systems in maternal health. Int J Gynecol Obstet 2016;134:13-17.

19 Alegana VA, Wright JA, Pentrina U, et al. Spatial modelling of healthcare utilisation for treatment of fever in Namibia. Int $\mathrm{J}$ Health Geogr 2012;11:6.

20 Bailey PE, Keyes EB, Parker C, et al. Using a GIS to model interventions to strengthen the emergency referral system for maternal and newborn health in Ethiopia. Int J Gynaecol Obstet 2011;115:300-9.

21 Makanga PT, Schuurman N, Sacoor C, et al. Seasonal variation in geographical access to maternal health services in regions of southern Mozambique. Int J Health Geogr 2017;16:1.

22 Ray N, Ebener S. AccessMod 3.0: computing geographic coverage and accessibility to health care services using anisotropic movement of patients. Int J Health Geogr 2008;7:63.

23 Black M, Ebener S, Aguilar PN. Using GIS to measure physical accessibility to health care. World Heal Organ 2004;3-4:1-22.

24 Ouma PO, Maina J, Thuranira PN, et al. Access to emergency hospital care provided by the public sector in sub-Saharan Africa in 2015: a geocoded inventory and spatial analysis. Lancet Glob Health 2018;6:e342-50.

25 Juran S, Broer PN, Klug SJ, et al. Geospatial mapping of access to timely essential surgery in sub-Saharan Africa. BMJ Glob Health 2018;3:e000875. 
26 Curtis A, Monet J-P, Brun M, et al. National optimisation of accessibility to emergency obstetrical and neonatal care in Togo: a geospatial analysis. BMJ Open 2021;11:1-10.

27 Oliphant NP, Ray N, Bensaid K, et al. Optimising geographical accessibility to primary health care: a geospatial analysis of community health posts and community health workers in niger. BMJ Glob Health 2021;6:1-11.

28 WHO. Referral systems: a summary of key processes to guide health services managers. Who, 2008: 1-9.

29 Kironji AG, Hodkinson P, de Ramirez SS, et al. Identifying barriers for out of hospital emergency care in low and low-middle income countries: a systematic review. BMC Health Serv Res 2018;18:291.

30 Manongi R, Mtei F, Mtove G, et al. Inpatient child mortality by travel time to hospital in a rural area of Tanzania. Trop Med Int Health 2014;19:555-62.

31 Portela MC, Lima SML, da Costa Reis LG, et al. Challenges to the improvement of obstetric care in maternity hospitals of a large Brazilian City: an exploratory qualitative approach on contextual issues. BMC Pregnancy Childbirth 2018;18:1-9.

32 Ibrahim A, Maximilien N, Lumbroso D. Emergency management of acute ischemic stroke. Intech 2013;32:137-44.

33 Hodder R, Lougheed MD, Rowe $\mathrm{BH}$, et al. Management of acute asthma in adults in the emergency department: nonventilatory management. CMAJ 2010;182:E55-67.

34 Ihantamalala FA, Herbreteau V, Revillion C. Improving geographical accessibility modeling for operational use by local health actors. Int $J$ Health Geogr 2020:1-15.

35 Cordier LF, Kalaris K, Rakotonanahary RJL, et al. Networks of care in rural Madagascar for achieving universal health coverage in Ifanadiana district. Health Syst Reform 2020;6:e1841437.

36 OpenStreetMap contributors. Data from: OpenStreetMap, 2019. https://wwwopenstreetmaporg/
37 HOT. Humanitarian OpenStreetMap team. Mapp. our world together, 2013. Available: https://wwwhotosmorg/

38 Shcherb V, Pelykh A, Mueller H. Offline mobile maps \& navigation, 2010. Available: https://osmandnet/

39 TAG-IP Company. Data from: TAG-IP, 2020. https://wwwtag-ipcom/

40 U.S. Departement of the Interior. U.S. geological survey, 2018. Available: https://wwwusgsgov/

41 NASA. Power project data sets, 2022. Available: https:// powerlarcnasagov/

42 NGO PIVOT. Data from: pivot Dashboard, 2020. http://pivotdashboardorg/authentification

43 R Core Team. R: a language and environment for statistical computing, 2019. Available: https://wwwr-projectorg/

44 Wigley AS, Tejedor-Garavito N, Alegana V, et al. Measuring the availability and geographical accessibility of maternal health services across sub-Saharan Africa. BMC Med 2020;18:1-10.

45 Moïsi JC, Nokes DJ, Gatakaa H, et al. Sensitivity of hospital-based surveillance for severe disease: a geographic information system analysis of access to care in Kilifi district, Kenya. Bull World Health Organ 2011;89:102-11.

46 Ashiagbor G, Ofori-Asenso R, Forkuo EK, et al. Measures of geographic accessibility to health care in the Ashanti region of Ghana. Sci African 2020;9:e00453.

47 Adams AM, Ahmed R, Ahmed S, et al. Modelling improved efficiency in healthcare referral systems for the urban poor using a georeferenced health facility data: the case of Sylhet City Corporation, Bangladesh. BMC Public Health 2020;20:1476.

48 Felix O, George A, Fredrick O, et al. The Cost-Implications of reaching universal coverage of maternity health services in Siaya County, Western Kenya. Public Health Res 2020;10:1-11.

49 Tanser F. Methodology for optimising location of new primary health care facilities in rural communities: a case study in KwaZulu-Natal, South Africa. J Epidemiol Community Health 2006;60:846-50. 